\title{
Swelling-Induced Folding in Confined Nanoscale Responsive Polymer Gels
}

\author{
Srikanth Singamaneni, ${ }^{\dagger}$ Michael E. McConney, ${ }^{\ddagger}$ and Vladimir V. Tsukruk* \\ School of Materials Science and Engineering, Georgia Institute of Technology, Atlanta, Georgia 30332. ${ }^{\dagger}$ Current address: Department of Mechanical, Aerospace and \\ Structural Engineering, Washington University, St. Louis, Missouri 63130. ${ }^{\ddagger}$ Current address: Materials and Manufacturing Directorate, Air Force Research Laboratory, \\ Wright-Patterson Air Force Base, Dayton, 0hio 45433-7702.
}

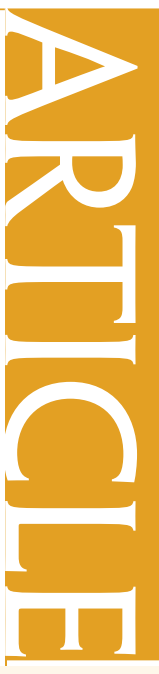

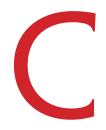
omplex 3D patterns with longrange uniformity is a universal phenomenon that is of interest in different fields including astronomy, geology, materials science, and biology. ${ }^{1-3}$ Folding of soft biomacromolecules, membranes, and tissues is an important process critical in such diverse phenomena as protein folding, skin wrinkling, and brain folding. Key examples of intricate periodic patterns observed in biological systems include phyllotaxis (arrangement of leaves in plants), wrinkling of the leaves, buckling of epithelial tissues under differential growth, ciliary folds of the eye, and folds of the brain and skin. ${ }^{4,5}$ Considering the role of mechanical force in biological morphogenesis, it has been suggested that mechanical and chemical processes can cooperate to provide feedback capable of accounting for complex phenomena such as scale invariance in biological morphogenesis. Folding is an essential event in biological pattern formation such as neurulation, which is involved in the folding of the notochord to form a neural tube, and the folding of the brain in developed animals to increase the surface area. ${ }^{6}$ However, the mechanics involved in the folding of compliant biological structures during growth in a confined environment remains elusive in many instances.

On the other hand, synthetic responsive compliant materials and structures, which can readily change their properties (optical, electrical, mechanical) or shape are extremely important for a wide variety of applications such as sensors, actuators, adaptive surfaces and interfaces, and drug delivery. ${ }^{7-14}$ Responsive synthetic compliant systems in the form of gel films, colloidal assemblies, and various interfacial strucwww.acsnano.org
ABSTRACT Mechanical instabilities such as buckling, wrinkling, creasing, and folding are commonplace in both natural and synthetic systems over a wide range of length scales. In this study, we focus on the spontaneous folding behavior of the highly swellable confined nanoscale (thickness below $100 \mathrm{~nm}$ ) gel films resulting in the formation of a network of regularly folded structures spontaneously emerging in the course of their swelling and drying. We suggest that regular self-folding is originated from periodic instabilities (wrinkles) caused by swellinginitiated stresses under confined conditions. Furthermore, folded gel structures can be organized into regular serpentine-like manner by imposing various boundary conditions on microimprinted surfaces. We suggest that this demonstration of uniform gel to mechanically mediate morphogenesis has far-reaching implications in the creation of complex, large-area, 3D gel nanostructures.

KEYWORDS: mechanical instabilities - polymer gels · spontaneous folding $\cdot$ responsive polymer $\cdot$ nanoimprinting

tures such as brushes, self-assembled monolayers, or block copolymers, which are sensitive to environmental conditions ( $\mathrm{pH}$, temperature, solvent, stress, light) can also be utilized to mimic the biological environment to gain a valuable insight into mechanistic aspects of morphogenesis. ${ }^{15-23}$ Moreover, water-swollen gel structures with extremely large swelling rates and abilities to form complex topographies are exploited as biomaterials for cell support, muscle-inspired actuation, and bioinspired sensing. ${ }^{24-27}$

Mechanical instabilities and organized wrinkling structures are frequently observed at multiple length scales in such compliant systems. ${ }^{28-33}$ Thin film buckling is a popular example of mechanically induced de novo pattern formation in a thin rigid material bound to a thick compliant substrate subjected to lateral compression. ${ }^{34-41}$ While mechanical instabilities have been extensively explored in thin rigid skins on soft under layers (e.g., metal coatings on polymers), they remain relatively underexplored and elusive in ultrathin, nanoscale gels firmly tethered to
*Address correspondence to vladimir@mse.gatech.edu.

Received for review December 22, 2009 and accepted March 08, 2010.

Published online March 16, 2010. $10.1021 /$ nn901886y

() 2010 American Chemical Society 


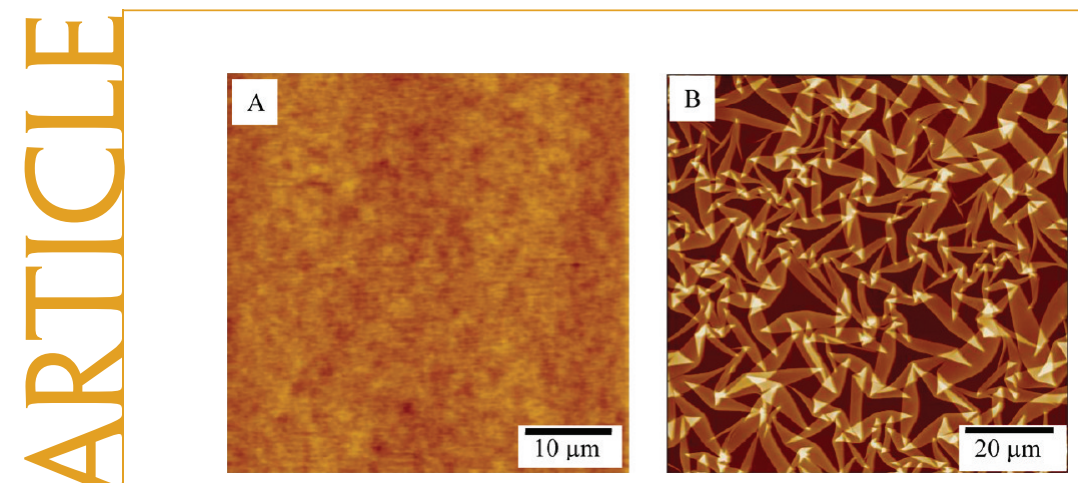

Figure 1. AFM image of (A) the spin-coated P2 VP film showing the smooth featureless surface morphology ( $Z$ scale, $5 \mathrm{~nm}$ ); (B) a large area of folded P2 VP film up on exposure to acidic ( $\mathrm{pH} 2.0)$ solution ( $Z$ scale, $400 \mathrm{~nm})$.

rigid surfaces. This is especially true for understanding their ability to initiate the formation of out-of-plane periodic structures and complex morphologies and under restrictive and periodic boundary conditions. ${ }^{42}$

Gels, swelling uniaxially under external stimuli exhibit smooth buckles and cusp-shaped singularities, which were related to and discussed in terms of linear elastic buckling. ${ }^{43}$ Various aspects of the buckling patterns in these structures have been extensively studied to reveal the temporal evolution and mechanistic aspects of the buckling phenomenon. ${ }^{44-48}$ It has been demonstrated that buckling and folding are not independent phenomena and wrinkles appear as a first order linear response to external or internal stresses. ${ }^{49}$ Folding and crumpling of compliant structures result in localization of the strain energy into a network of ridges and sharp corners. ${ }^{50-52}$

Herein, we report ultrathin $(20-100 \mathrm{~nm})$ gel films tethered to solid substrates which exhibit unusual spontaneous and regular self-folding under swelling induced compressive stresses. In this system, the anchoring of the gel nanofilm to a stiff substrate constrains swelling and frustrates the buckling deformation in the course of swelling, leading to localized pinched folds and eventually to anisotropic folded nanosheets at multiple scale lengths. Spontaneous self-folding of tethered nanoscale gel films occurs in the course of their swelling followed by drying. In our recent publication, we suggested that regular self-folding is originated from periodic instabilities (wrinkles) caused by swellinginitiated stresses. ${ }^{53}$ In this paper, we further expand this intriguing phenomenon and demonstrate for the first time that folded gel structures can be organized into long-range regular serpentine-like topologies by imposing various boundary conditions via microprinted surfaces, which might have far-reaching implications in the creation of complex artificial 3D gel nanostructures.

\section{RESULTS AND DISCUSSION}

Responsive Gel Films. In this work we employed poly-2vinylpyridine (P2 VP) to form nanoscale gel films tethered to a solid substrate. As known, P2 VP is a weak cationic polymer that exhibits dramatic globule-coil transformation due to the protonation of the pyridine group below pH 4.0.54-56 As a result of this transition, a significant and fast swelling might occur under proper solution conditions.

Cross-linked $90 \mathrm{~nm}$ thick P2 VP gel film grafted onto a silicon wafer with a native oxide layer of $\sim 1 \mathrm{~nm}$ shows uniform and smooth surface morphology with local microroughness below $1 \mathrm{~nm}$ (Figure 1A). P2 VP exhibits strong interaction with the silicon oxide surface owing to the hydrogen bonding between the surface hydroxyl groups and nitrogen on the pyridine ring. ${ }^{57} \mathrm{In}$ the protonated state (below $\mathrm{pH} 4.0$ ), the electrostatic interaction between the positively charged pyridine units and negatively charged silicon oxide surface further promotes strong anchoring to the surface. Surprisingly, simple exposure of the cross-linked gel films to $\mathrm{pH} 2.0$ solution resulted in the transformation of the initially smooth morphology into a network of highly anisotropic structures, which is a subject of the current in-depth study (Figure 1B).

Topology of the Folded Structures. Careful inspection of surface morphology of these anisotropic structures revealed that the observed structures are indeed regular folds in the gel film. As we observed, each of the fold structure comprises two distinct regions, the hinge and the limb, with slightly different thicknesses as depicted by the cross-section of the AFM image (inset of Figure $2 \mathrm{~A})$. While the hinge side is one straight edge connecting the nodes, the limb side is composed of either one or two vertices connected by straight ridges.

Each fold starts and ends in a sharp corner with an angle of $\sim 30^{\circ}$, which in most of the cases overlaps with an adjacent fold. The overlapping sharp corner of the double fold aligns to the vertex of the other fold's limb, indicating that the fold collision resulted in an inhibitory effect on the pinched buckle growth. The folded film exhibits surfaces with three distinct heights as depicted by the cross sections in Figure 2. The three distinct surfaces correspond to the stretched residual base gel layer tethered to the substrate (thickness of 22 $\mathrm{nm}$ ), single folded region ( $\sim 120 \mathrm{~nm}$ thick), and region corresponding to double folds ( $\sim 250 \mathrm{~nm}$ thick).

We suggest that the spontaneous formation of folds is caused by a competition between stress developed upon swelling and the boundary constraints similar to that observed for bulk gel structures. ${ }^{47,48}$ Indeed, it is well-known from the bulk structures ( $\mathrm{mm}$ scale thick structures) that swollen gel constrained at the film-substrate interface is subjected to a gradient compressive stress, which decreases from the topmost surface to the bottom. ${ }^{43}$ While the gradient in compressive stress results in smooth sinusoidal buckles in the bulk gels, the same phenomenon in nanoscale thick gel, investigated here, resulted in buckling deformation as the way to release localized stresses. This initial buckling is immediately followed by localization of the deformation into a network of nonsinusoidal pinched buckles 

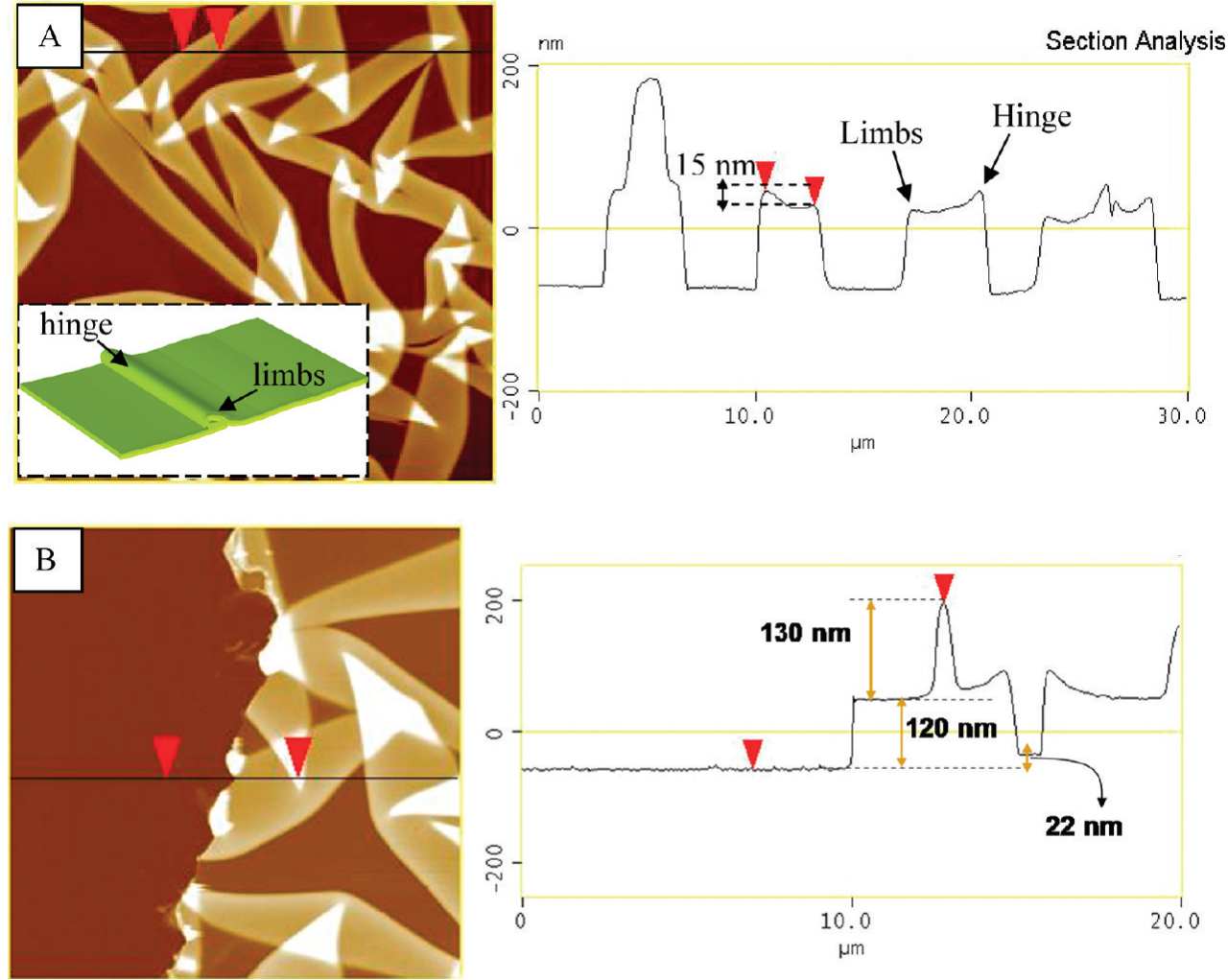

Figure 2. AFM image and cross-section (A) along the folded structure showing the topography the fold (inset shows the schematic representation of the folding of the P2 VP film); (B) along a scratch showing the thickness of surface bound layer, single and double folds.

expanded across large surface areas. Pinched surface buckles were observed at the outermost rim of the wet surface, which was only briefly exposed as has been described elsewhere. ${ }^{53}$

As the swelling proceeds further, the pinched surface buckles meander and meet, thereby forming nodes. During this stage, the gel nanofilm goes through massive transformations leading to micrometer scale lenticular surface structures due to strong shear forces at the film-substrate interface. The growth of the folded structures is arrested when the surrounding regions of the residual gel surface layer become too thin $(\sim 20 \mathrm{~nm}$ ) to further shear (due to the strong anchoring to the substrate). ${ }^{53}$ The folded structures evolve via a series of fast and discrete "popping" steps. The intersection points guide the drying-induced folding and sudden release from substrate, which is evident by the majority of the intersections lying on top of the hinge side instead and not underneath the hinge-side of the buckle.

The confinement of the ultrathin gel films caused by strong adhesion to the substrate and high degree of swelling of the loosely cross-linked gel film on the top are important for inducing these unique folding patterns. As was mentioned above, P2 VP films exhibit strong interaction with the silicon oxide owing to the combined effect of hydrogen bonding and electrostatic interactions. To test the role of this bonding, P2 VP gel was de- posited on a silicon wafer precoated with positively charged polyallyl amine hydrochloride (PAH), which removes hydrogen bonding and introduces Coulombic repulsion between protonated pyridine groups and the substrate. For this control system with greatly reduced interfacial strength, exposure of the gel films to acidic conditions at $\mathrm{pH} 2.0$ resulted in complete delamination of the P2 VP layer with no folding phenomenon in contrast to the previous case. This difference suggests the critical role of strong adhesion between the gel nanofilm and the substrate in self-folding phenomenon.

On the other hand, high compliance of gel nanofilms plays an important role in the phenomenon ob-
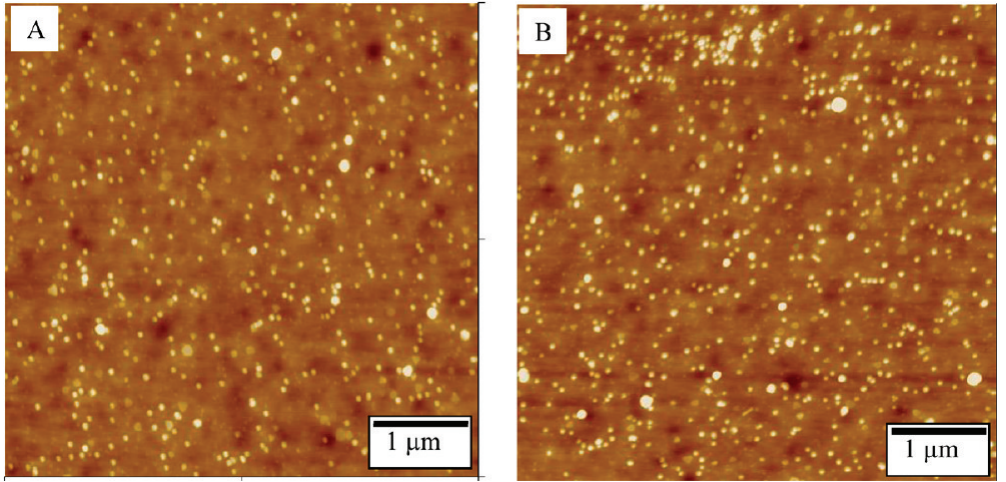

Figure 3. AFM images of (A) the P2 VP film imbedded (by in situ reduction) with gold nanoparticles; (B) exposure to $\mathrm{pH} 2.0$ showing no discernible changes in morphology, thereby indicating the importance of swellability in the buckling and folding process ( $Z$ scale, $40 \mathrm{~nm}$ ).

VOL. 4 - NO. $4 \cdot 2327-2337 \cdot 2010$ 

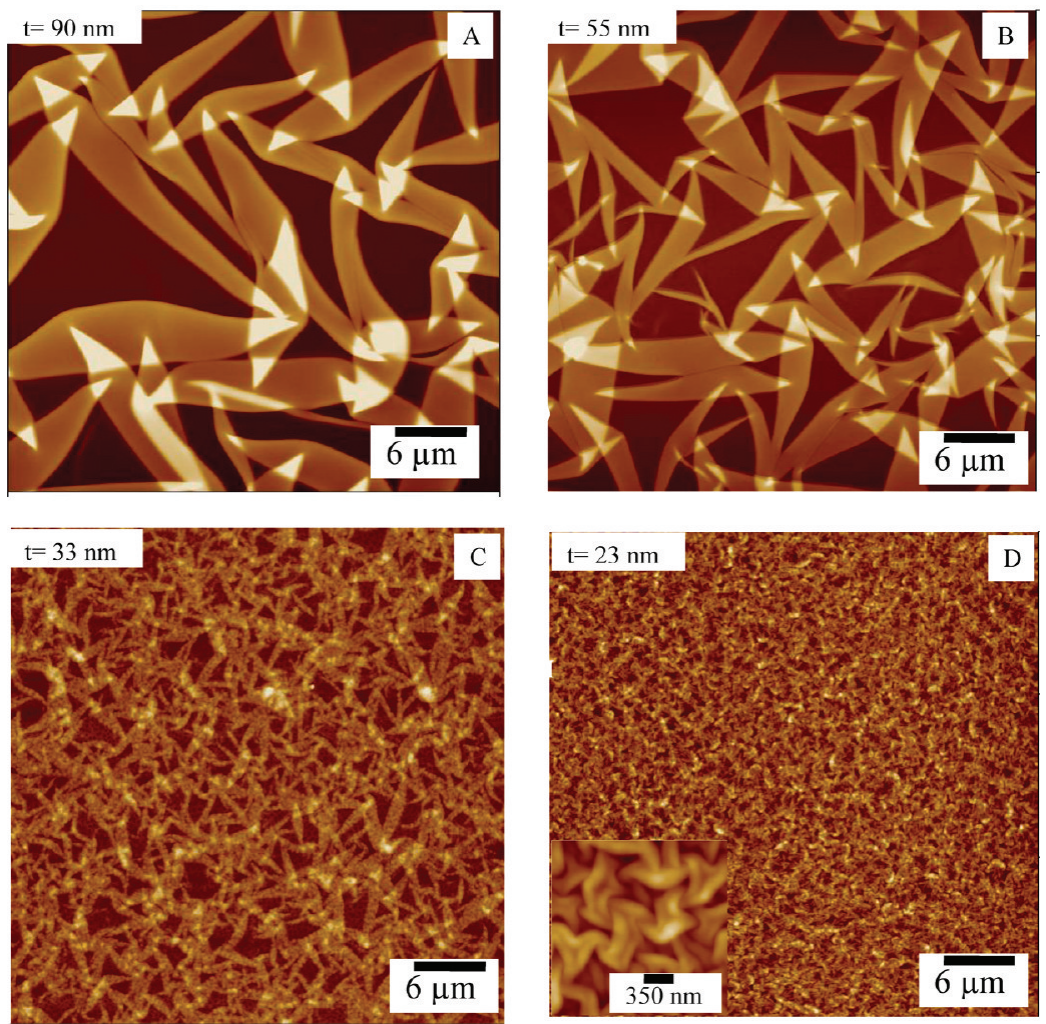

Figure 4. AFM images showing the folding in P2 VP films with thickness (A) 90 (Z scale, 300); (B) 55 (Z scale, 200); (C) 33 ( $Z$ scale, 150); and (D) $23 \mathrm{~nm}$ (Z scale, $50 \mathrm{~nm}$ ).

served. Indeed, we observed that additional stiffening of gel nanofilms might suppress their ability to swell, buckle, and fold. To stiffen the gel nanofilm, we conducted in situ reduction of metal nanoparticles inside these films by exposing this film to gold salt $\left(\mathrm{HAuCl}_{4}\right)$ followed by reduction using sodium citrate according to the known approach. ${ }^{58}$ This treatment did not result in any noticeable changes in the initial morphology of the gel film except for the presence of well dispersed nanoparticles (Figure 3). The presence of these nanoparticles should significantly increase the stiffness of the gel films as was demonstrated earlier for similar films. ${ }^{59}$ This film stiffening and the reduction of their swelling ability completely suppressed the folding phenomenon thus confirming that high compliance combined with high swelling are both critical for the folding phenomenon observed here.

To further understand the origin of the observed lenticular folding patterns, we considered how the conditions for buckling patterns in swollen gels attached to the surface can initiate such a phenomenon. As discussed by Tanaka and co-workers, minimization of the potential energy of film with thickness $h$ and elastic modulus $E_{\mathrm{o}}$ tethered to the substrate with the elastic modulus $E_{2}$ leads to the condition for the first buckling mode, $\lambda_{c}$, given by

$$
\frac{2 \pi}{\lambda_{c}}=\left(\frac{E_{0}}{E_{2}}\right)^{1 / 4} \frac{\sqrt{3}}{h}
$$

The elastic modulus $\left(E_{\mathrm{o}}\right)$ of the films in the swollen state was measured to be $600 \mathrm{kPa}$ using colloidal probe nanomechanical measurements as discussed in detail elsewhere. ${ }^{59}$ Taking the modulus $\left(E_{2}\right)$ of the solid P2 VP film as about $2 \mathrm{GPa}$ (glassy state) and using eq 1, the wavelength of the buckling pattern formed at the equilibrium swollen state was estimated to be around 3 $\mu \mathrm{m}$. This value is close to the width of the folded structures observed here $(2.8 \mu \mathrm{m})$ indicating that the folding can originate from stress-induced periodic wrinkles, thus preserving the original characteristics (wavelength and thickness dependence).

Dimensions of the Folded Structures. The folds discussed so far for a film with a thickness of $90 \mathrm{~nm}$ are about 3 $\mu \mathrm{m}$ wide and a few tens of micrometers long. The folding dimensions can be scaled down to a submicrometer dimension by decreasing the initial gel film thickness down to $20 \mathrm{~nm}$. With the decrease in the thickness of the film, the length and the width of the folds decrease, while surface density of the folds increases dramatically (Figure 4). However, the characteristic shape of the folds remains the same while the dimensions and the density of the folded structures monotonously change with initial film thickness (Figure 5).

In fact, the histograms of the length and the width distribution clearly reveal the change in the length and the width of the folds with the gel film thickness (Figure $5 A-D)$. The length and width of the folded sheets linearly increase with the thickness of the gel film (Fig- 


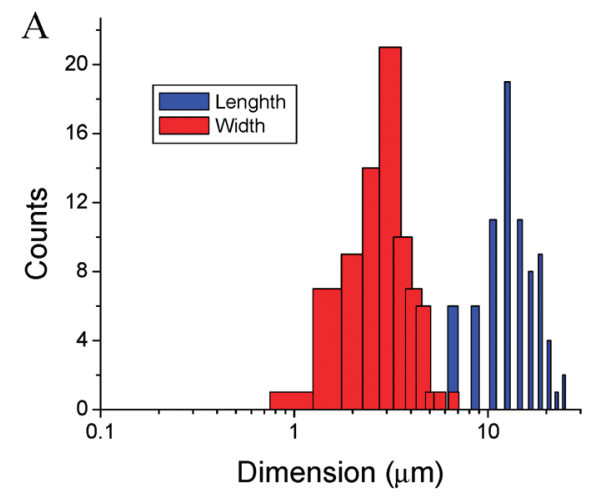

C

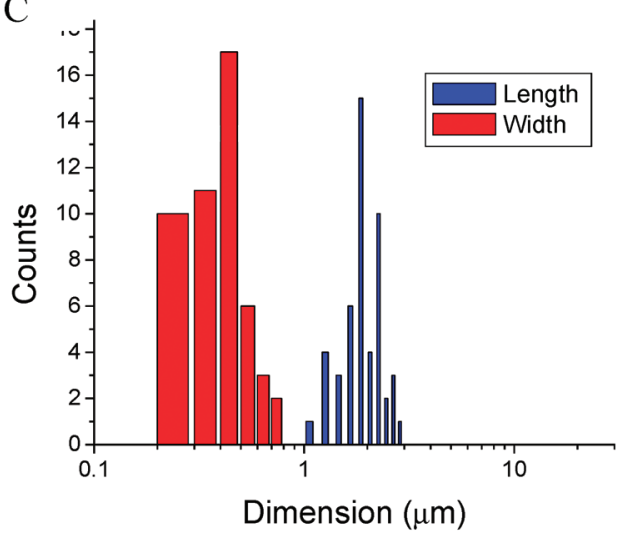

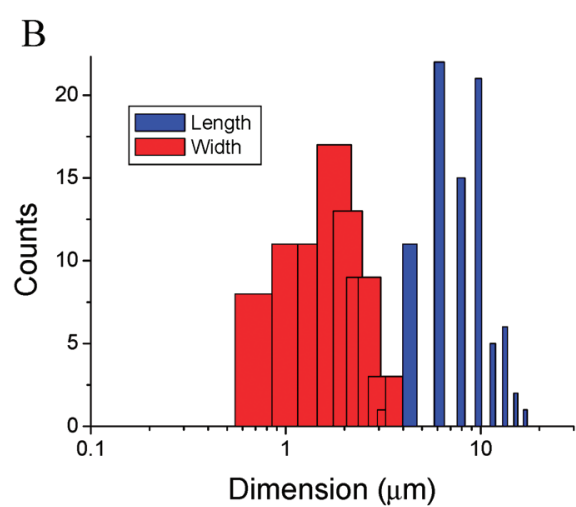

D

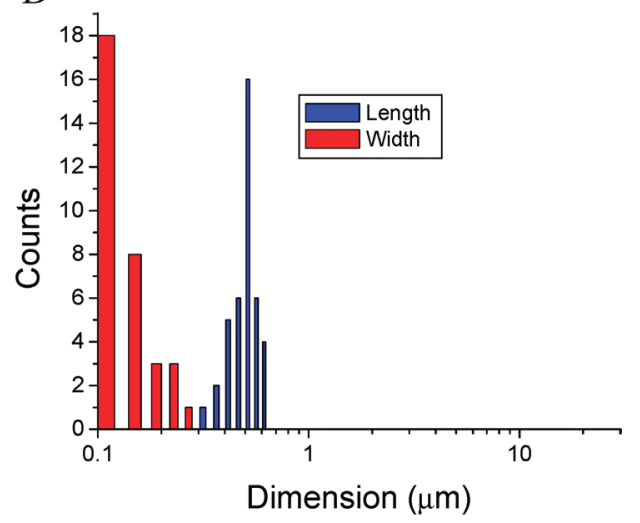

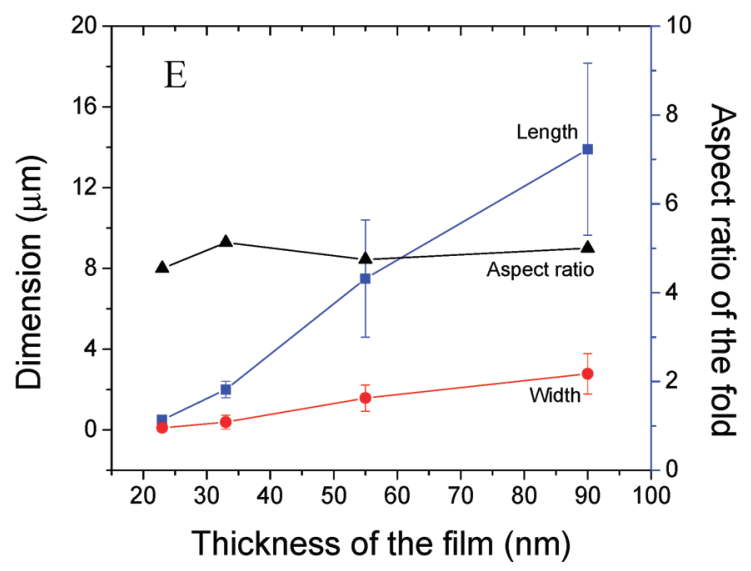

Figure 5. Histograms showing the length and width distribution of the folds in the P2 VP films with thickness (A) 90 , (B) 55, (C) 33 , and (D) $23 \mathrm{~nm}$. (E) Plot showing the linear scaling of the average length and the width of the folds with the thickness of the film. The aspect ratio of the folds remains close to $5 \pm 0.2$ over the entire range of film thicknesses studied here.

ure $5 \mathrm{E}$ ). This change holds over an order of magnitude change in the length of the folds, $14 \mu \mathrm{m}$ for $90 \mathrm{~nm}$ thick film to $500 \mathrm{~nm}$ for $23 \mathrm{~nm}$ thick film. On the other hand, the overall shape (aspect ratio (length to width ratio), ridges, vertices, and corners) of the folding structures is much less affected by the initial thickness. Indeed, the aspect ratio remains remarkably constant within $5 \pm 0.2$ for all gel morphologies generated here (Figure 5E). This self-regulation of the size and shape is quite surprising, essentially indicating a consistent mechanicalbased intrinsic folding mechanism.

Topology of Folding Structures and Influence of Boundary Conditions. To monitor the deformation of the gel film (local strains) during the folding process and the influence of www.acsnano.org boundary conditions on substrates, the P2 VP films were imprinted with periodic arrays of stripes and posts with

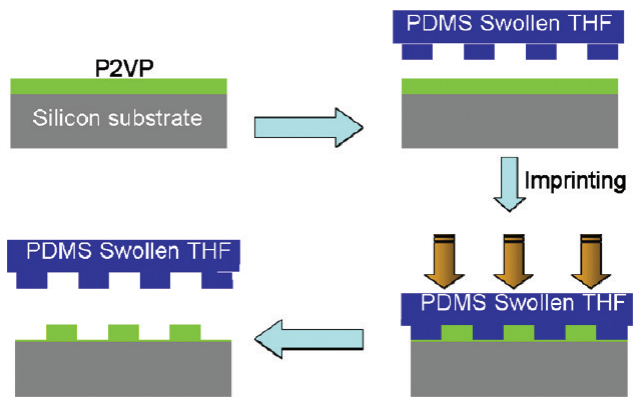

Figure 6. Schematic showing the imprinting technique to create the film with nanostripe pattern, nanopost pattern, and one-dimensional structures in the P2 VP film. 

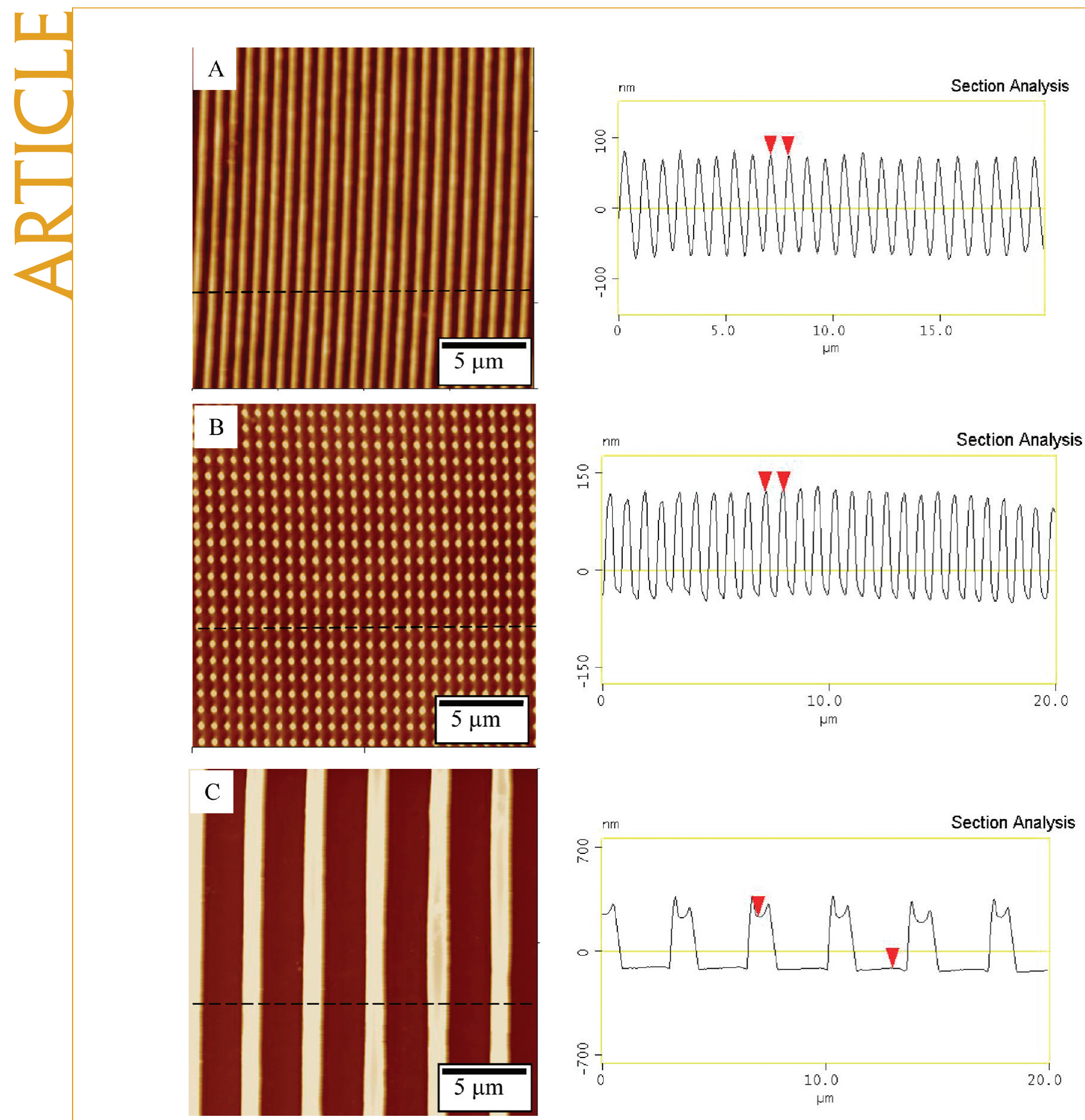

Figure 7. AFM images of (A) nanostripe pattern with a periodicity of $900 \mathrm{~nm}$; (B) a square lattice of nanopost pattern with a periodicity of $900 \mathrm{~nm}$, which act as internal rulers to monitor the subsequent deformation of the film; (C) one-dimensional P2 VP structures with a periodicity of $3 \mu \mathrm{m}$. Also shown are the corresponding cross-sections showing the nanostripe pattern imprinted into the P2 VP film that serve as a boundary condition for the swelling system.

predetermined microscopic spacing (see Figure 6 and Methods section). The nanostripe and nanopost patterns with predetermined periodicity can act as an internal ruler to monitor the strain and thus structural changes, whereas the larger scale microstripe pattern results in a one-dimensional confinement of P2 VP gel, thus imposing additional lateral boundary conditions.

Three different periodic patterns, namely nanostripe, nanopost, and microstripe patterns, were im- printed into the P2 VP film with a thickness of $90 \mathrm{~nm}$ according to the usual procedure (Figure 7).$^{60}$ Figure 7 shows the AFM images of various patterns and the corresponding crossections. AFM image of the P2 VP film imprinted with the stripe pattern with a periodicity of $900 \mathrm{~nm}$ is shown in Figure 7A. The cross-section shown in the image clearly depicts the long-range uniformity of the stripe pattern. Similarly, the nanopost pattern comprises a 2D square array of nanoposts with a peri- 
odicity of $900 \mathrm{~nm}$ as well (Figure 7B). The cross-section of the image shows the uniform height of the nanoposts imprinted into the P2 VP film. Finally, the microstripe pattern resulted in well-separated (by $1.7 \mu \mathrm{m}$ ) one-dimensional P2 VP structures with a width of 1.3 $\mu \mathrm{m}$ for a total periodicity of $3 \mu \mathrm{m}$ (Figure $7 C$ ).

Upon exposure to acidic conditions, the P2 VP film with stripe and the nanopost patterns (with a periodicity of $900 \mathrm{~nm}$ ) exhibited organized lenticular structures popping up from nanoimprinted film similar to that observed in a uniform flat film (Figure 8). The folded film with nanostripe pattern exhibits a complex network of helices interconnected by a network of stripes, highlighting the complex topology of the folded film (Figure $8 \mathrm{~A}$ ). The helical structures in the image correspond to the individual folds, while the network of lines connecting them corresponds to the base layers described above. Similar regions (folded and base layers) can be indentified in the folded film with the imprinted nanopost pattern. These observations establish the similarity of the folding procedure in the smooth and imprinted films. However, the presence of the imprinted patterns with known initial spacing can be utilized to monitor the deformation in the folded gel film by using the micropattern as an internal ruler, which allows monitoring the localized strains.

Indeed, the periodicity of the embedded regular lattices increased from $900 \mathrm{~nm}$ in the initial undeformed gel film to $\sim 1800 \mathrm{~nm}$ in the folded film indicating remarkably large $(\sim 100 \%)$ tensile strain arising in the course of swelling and folding (Figure 9). The large strain in the gel film should result in the significant plastic deformation of the swollen hydrogel structure thus "freezing" mechanical instabilities after drying. The stretching and thinning of the nanoscale gel film indicate that these interbuckle regions provide the material for the buckle growth and are eventually depleted enough to inhibit further buckling.

The embedded patterns also enable the tracking of the folding behavior and demonstrate lateral shearing and stretching of the swollen tethered gel film (Figure $9 \mathrm{~B})$. This way, we observed that the contour of the nanostripes in the helix underscores the asymmetry of the folded structure with the curvature of the helices different on either side of the folds. Furthermore, in the case of the square lattice structure, shearing results in the transformation of the pattern to a hexagonal lattice indicating strong asymmetric in-plane shearing in the course of folding (Figure 9D). Thus, the internal rulers in the form of stripe and nanopost patterns clearly reveal large, 100\%, in-plane strain and the complex shearing topology of the folded structures.

Long-Range Confined Folded Structures. The importance of long-range effects in the deformation mode was demonstrated using the larger scale pattern ( $3 \mu \mathrm{m}$ periodicity). We like to point out that the $3 \mu \mathrm{m}$ periodicity chosen here is close to the expected buckling wave-
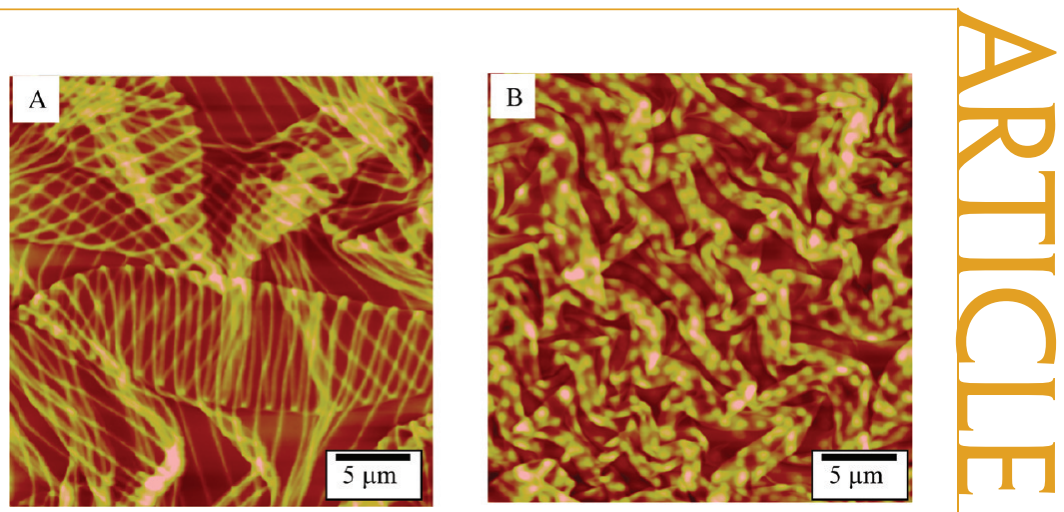

Figure 8. AFM images of the folded structure of the film depicting the lenticular shape of the folded structure with (a) nanostripe pattern ( $Z$ scale: 400 $\mathrm{nm}$ ) (B) nanopost pattern (Z scale: $300 \mathrm{~nm})$.

length and the observed $2.8 \mu \mathrm{m}$ fold width. Interestingly, imposing this lateral constraint resulted in the inhibition of complete folding and the formation of individual, localized, ordered folded structures (Figure 10). Exposure of the patterned sample to acidic solution at $\mathrm{pH} 2.0$ resulted in a dramatic transformation of the initial gel nanofilm to uniformly oriented, continuous "telephone-cord" structures (Figure 10). A closer observation of the AFM images and the cross-section analysis clearly reveals that the telephone cord structure is indeed periodic buckles transformed into twisted folds (Figure 10 B,C). Overall, the structure represents a chiral array with right-handed deformed helical structures which repeat themselves across macroscopic surface areas. Moreover, wider spaced patterned samples (to 7 $\mu \mathrm{m}$, which is more than twice the width of the folds in flat films) resulted in the misalignment and randomiza-
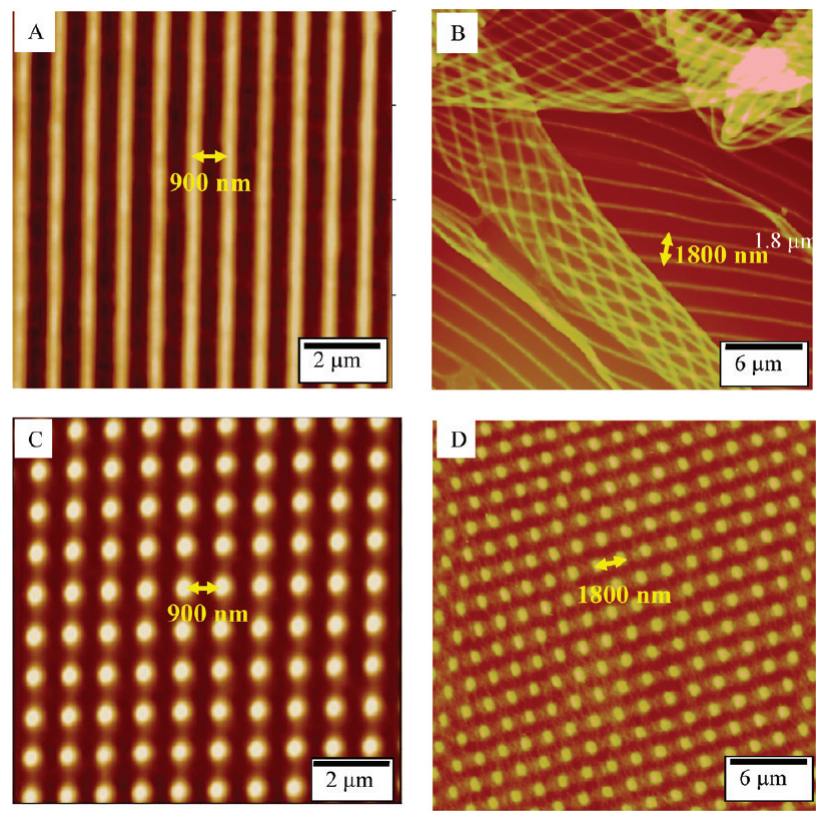

Figure 9. Higher magnification topography showing (A) pristine nanostripe pattern; (B) helical morphology of a single fold in the film exposed to $\mathrm{pH}$ 2.0, highlighting the strain in the folded structure; (C) pristine nanopost pattern; and (D) transformed hexagonal lattice structure due to the lateral shear strain in the folding process. 


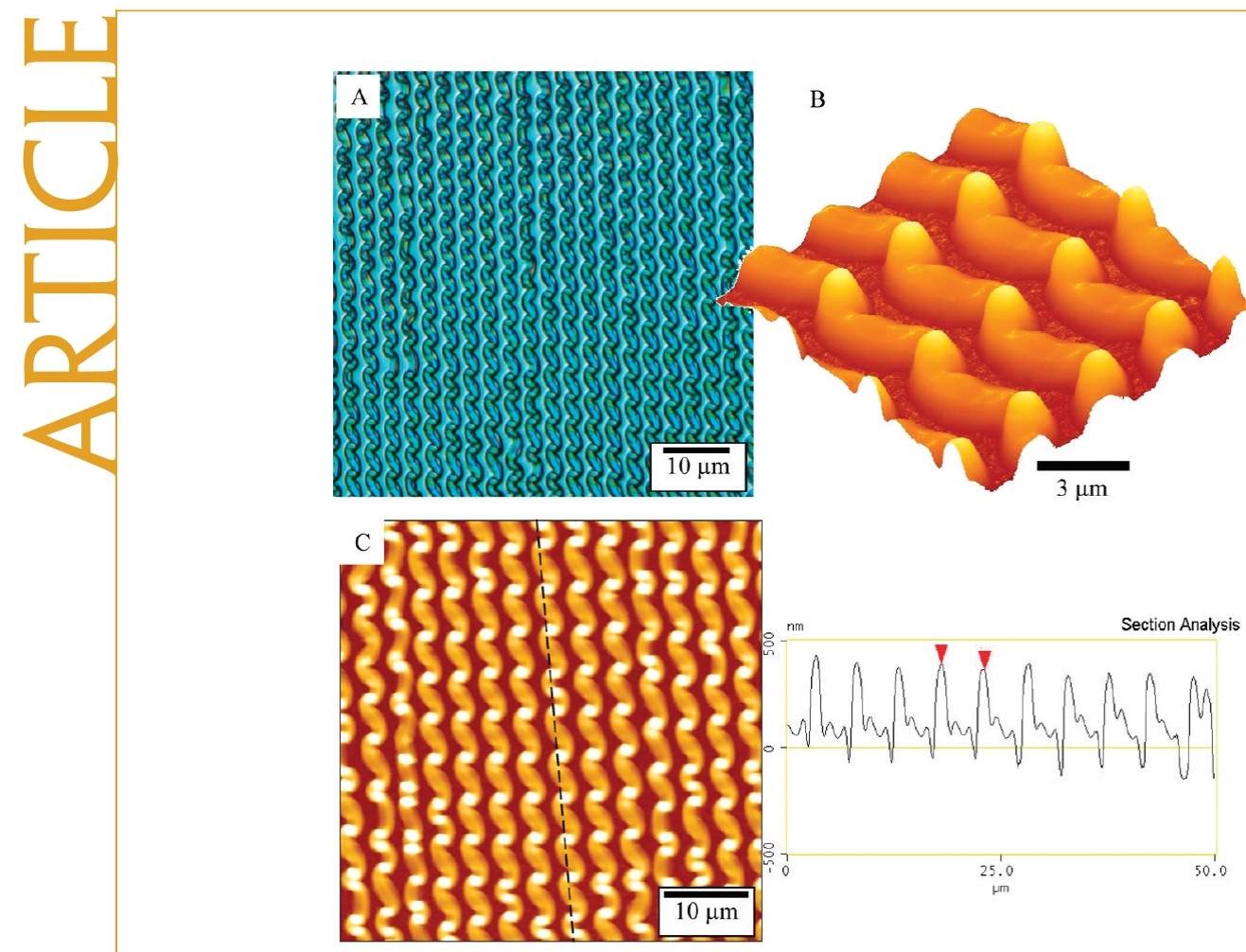

Figure 10. (A) Optical image showing the remarkable long-range uniformity of the folds along the length of the folds and (B) 3D AFM image of the folding. (C) Cross-section along the folded structure showing the three discrete heights of the fold regions, the intermediate regions, and the substrate-grafted layer.

tion of the serpentine structures in stark contrast to the previous case (Figure 11).

We suggest that the spatial alignment of the folded structures comes from the stress-strain gradient across the thinner regions in between the tall stripes. In other words, the limited supply of material between the growing structures creates long-range spatial alignment of the features through local growth competition, which causes the film to self-regulate and avoid complete separation from the substrate such as that observed for flat films. These results indicate that preexisting topological patterns acting as boundary conditions for mechanical stress-strain systems can reinforce and develop new topographical self-folded patterns.
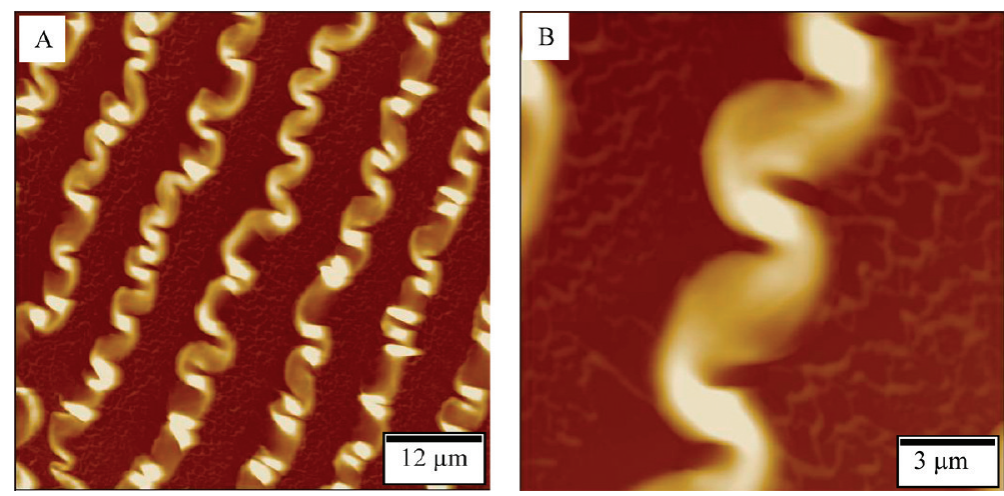

Figure 11. AFM image (A) showing the lack of long-range order in the folded structure when the individual one-dimensional structures are separated by larger spacing $(7 \mu \mathrm{m})$; (B) higher magnification image showing that the folding topology remains similar to that observed in Figure 10.
Apart from obtaining uniform folding patterns over large areas of the films, the patterns can be localized to predetermined regions by localized exposure of the film to acidic conditions. To demonstrate the confinement of the folding patterns to predetermined regions, we employed capillary transfer lithography to deposit a polystyrene mask on the cross-linked P2 VP film (as described in the Methods section). ${ }^{61}$ Figure $12 \mathrm{~A}$ shows the schematic representation of the steps involved in confining the folding patterns to localized regions of the P2 VP film. The process involved the deposition of a periodic polystyrene pattern $(3 \mu \mathrm{m}$ periodicity) followed by exposure to $\mathrm{pH} 2.0$ solution and subsequent removal of polystyrene (dissolving in toluene).

The AFM images of the P2 VP gel film swollen on these substrates exhibit a uniform array of folded regions interleaved with pristine regions (Figure 12B,C). The higher magnification AFM image clearly reveals that the folding is confined with a high degree of fidelity to the original polystyrene mask. Furthermore, it can be clearly observed that the folded regions contain areas that are higher (folds) and lower (surface bound layer) compared to the pristine regions in complete agreement with the previous suggestion. Thus, confinement of the folding patterns clearly demonstrates the localization of the swelling-mediated mechanical stresses and the possibility of fine control of the surface features that can be achieved across macroscopically significant surface areas.

\section{CONCLUSIONS}

We have demonstrated the formation of regular folding patterns in an ultrathin $\mathrm{pH}$ responsive polymer gel bound to a rigid substrate. Furthermore, we have demonstrated that simple boundary conditions imposed onto the nanoscale gel film can lead to intriguing structures (such as telephone cord buckles) and spatial confinement of the folds to predetermined regions. The unique capabilities of the purely mechanical-based pattern formation demonstrated in this simple gel may provide insight into the 
role of mechanics in biological pattern formation. The results demonstrate that de novo pattern formation via controlled mechanical stress can form discrete highaspect folded structures with uniform feature sizes and scale-invariance. We suggest that the uniform sinusoidal buckles are insufficient to relieve the stress in nanoscale gel films causing the stress to localize into pinched buckles, which finally fold. The material system investigated here is in sharp contrast to conventional systems studied to date in that it is composed of an nanoscale compliant gel material tethered to rigid substrate. The large volumetric expansion of the swollen tethered gel films forces the system to explore modes beyond simple linear elastic buckling and into an out-of-plane deformation mode.

Moreover, the stress distribution under confined conditions on micropatterned surfaces can be used to form organized folded structures, while simultaneously maintaining and effecting the overall pattern scale and alignment. It is interesting to note that the folds in these gel structures bear remarkable similarity to the folds observed in the developed brain of mammals, suggesting mechanical stress - strain systems during the growth processes. ${ }^{62}$ Furthermore, recent studies have provided strong evidence that the new growth of plant buds is highly affected by local stress - strain fields, which highlights the importance of stress-strain mechanics in pattern formation of swollen gels. ${ }^{63,64}$ In light of these findings, we sug-
A
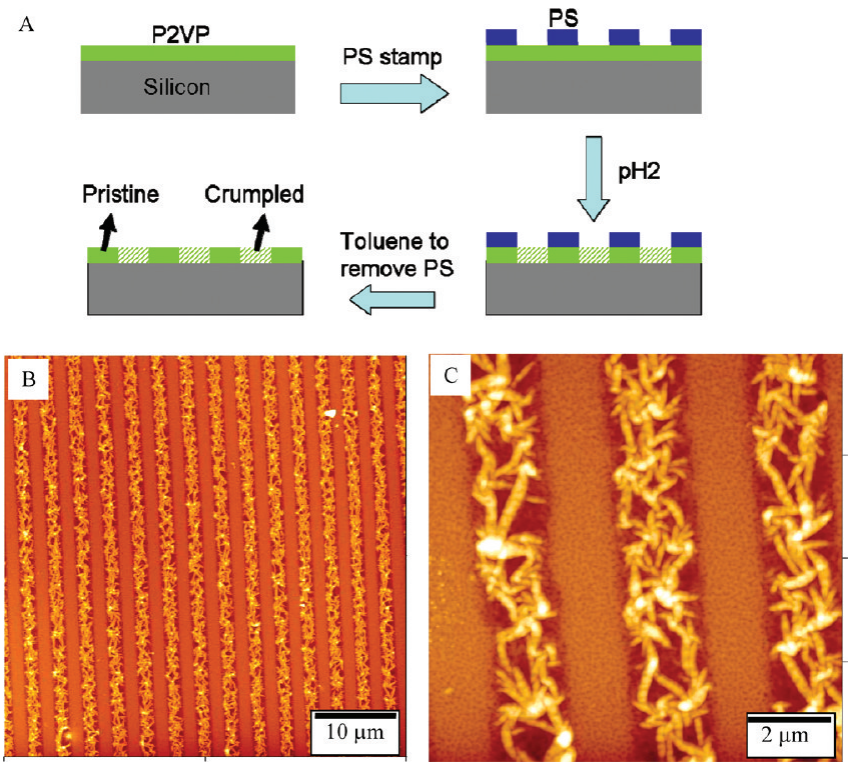

Figure 12. (A) Schematic showing the process for confining the folding to predetermined regions using capillary transfer lithography to deposit a PS pattern on the P2 VP film. (B,C) AFM images showing the confinement of the folds: (B) large scale image showing the periodically interleaving of the folded and unfolded regions ( $Z$ scale, $150 \mathrm{~nm}$ ); (C) higher magnification image showing the sharp boundaries between the folded and pristine regions ( $Z$ scale, $100 \mathrm{~nm}$ ).

gest that this demonstration has far-reaching implications in the creation of complex, large-area, 3D gel nanostructures and serves as a model system for understanding the mechanically mediated morphogenesis.

\section{METHODS}

P2 VP Gel Films. The ultrathin P2 VP films were prepared as follows: $0.2 \mathrm{~g}$ of P2 VP $\left(M_{\mathrm{w}}=144 \mathrm{~kg} / \mathrm{mol}\right.$, Polymer Source, Inc.) and $0.2 \mathrm{~mL}$ of 1,4-diodobutane (DIB) were dissolved in a $10 \mathrm{~mL}$ solvent mixture comprising $9 \mathrm{~mL}$ of nitromethane (NM) and $1 \mathrm{~mL}$ of tetrahydrofuran (THF). The resulting solution in a closed scintillation vial was kept at $60^{\circ} \mathrm{C}$ (in an oil bath) for $2 \mathrm{~h}$ with magnetic stirring to accelerate the quarternization reaction between P2 VP and DIB. In the course of the reaction, DIB molecules attach mainly by one end enabling the chains to cross-link and form a gel. To alter the thickness of the PVP films, the quaternized 2\% P2 VP solution (described above) was diluted by adding the solvent mixture ( 9 parts of NM and 1 part THF) to prepare $1 \%, 0.66 \%$, and $0.5 \%$ solutions. The P2 VP films were exposed to aqueous $\mathrm{pH} 2.0$ solution (nanopure water adjusted by $\mathrm{HCl}$ ).

Silicon wafers cut to a typical size of $1 \times 2 \mathrm{~cm}$ were cleaned in a piranha solution [1:3 (v/v) $\left.\mathrm{H}_{2} \mathrm{SO}_{4} / \mathrm{H}_{2} \mathrm{O}_{2}\right]$, according to a usual procedure adapted in our laboratory. ${ }^{65}$ Attention: Piranha solution is extremely dangerous and should be handled very carefully. Silicon wafers of the $\{100\}$ orientation with one side polished (Semiconductor Processing Co.) were atomically smooth (microroughness below $0.2 \mathrm{~nm}$ ). After cleaning, the substrates were then rinsed thoroughly with nanopure water and dried with dry nitrogen before they were used. Spin coating was employed to deposit thin films P2 VP on the clean silicon substrate. The P2 VP films were cross-linked and tethered to the substrate by annealing at an elevated temperature of $120^{\circ} \mathrm{C}$ for $2 \mathrm{~h}$.

Nanoimprinting of P2 VP Films. For monitoring the deformation of the P2 VP film, stripe pattern and square lattice of micropost structures were imprinted into the film (Figure 7). A polydimethyl siloxane (PDMS) stamp with a negative replica of nanopost (square array of nanopore arrays) was fabricated using a SU8 master fabricated by interference lithography. ${ }^{66}$ For nanoimprinting the PDMS stamp was swollen in THF and was brought into conformal contact with the P2 VP film and compressed for $1 \mathrm{~min}$. The THF in the PDMS stamp softens the P2 VP film casting a faithful replication of the nanopost structure in the P2 VP gel film. The imprinted P2 VP films were cross-linked by annealing at $120^{\circ} \mathrm{C}$ for $2 \mathrm{~h}$.

Capillary Transfer Lithography. To localize and pattern the folding, patterns of polystyrene were employed as a physical mask on the P2 VP film to prevent the $\mathrm{pH} 2.0$ exposure in the masked regions. Polystyrene (with $3 \mu \mathrm{m}$ periodicity) was deposited using capillary transfer lithography $(C T L)$ illustrated in Figure 12A. ${ }^{61}$ In brief, a PDMS stamp (with 10-mm periodicity) was soaked in toluene for 1-2 min and brought into conformal contact with the polystyrene film on the PDMS substrate (swollen in toluene) and pressed for $1 \mathrm{~min}$. The polystyrene infiltrated the receding portions of the PDMS stamp by capillary action. The pattern was then transferred onto the P2 VP surface by contact of the PDMS stamp for $1 \mathrm{~min}$.

Atomic Force Microscopy. The surface morphology of the gel films was studied with a Dimension 3000 atomic force microscope (AFM) in a light tapping mode according to the procedure adapted in our lab. ${ }^{67}$ The thickness of the films were measured by AFM scratch test in which AFM scanning was performed along the edge of a scratch made in the film using a fine needle. The cross-section histograms (bearing analysis) were employed to measure the thickness of the film.

Acknowledgment. The work is supported by AFOSR and AFRL, FA9550-09-1-0162 project. The authors would like to thank S. Chang and S. L. Young for technical assistance and M. Gupta for useful discussions. 
Supporting Information Available: Real-time video showing the formation of the folds upon exposure of the responsive polymer film to acidic solution. This material is available free of charge via the Internet at http://pubs.acs.org.

\section{REFERENCES AND NOTES}

1. Levin, S. A. The Problem of Pattern and Scale in Ecology. Ecology 1992, 73, 1943-1967.

2. Cross, M. C.; Hohenberg, P. C. Pattern-Formation outside of Equilibrium. Rev. Mod. Phys. 1993, 65, 851-1112.

3. Hayashi, T.; Carthew, R. W. Surface Mechanics Mediate Pattern Formation in the Developing Retina. Nature 2004, 431, 647-652.

4. Rossomando, E. F.; Alexander, S. Morphogenesis: An Analysis of the Development of Biological Form; Marcel Dekker Inc.: New York, 1992.

5. Smith, R. S.; Guyomarch, S.; Mandel, T.; Reinhardt, D.; Kuhlemeier, C.; Prusinkiewicz, P. A Plausible Model of Phyllotaxis. Proc. Natl. Acad. Sci. U.S.A. 2006, 103, 1301-1306.

6. Bard, J. Morphogenesis: The Cellular and Molecular Processes of Developmental Anatomy; Cambridge University Press: New York, 1990.

7. Luzinov, l.; Minko, S.; Tsukruk, V. V. Adaptive and Responsive Surfaces through Controlled Reorganization of Interfacial Polymer Layers. Prog. Polym. Sci. 2004, 29, 635-698.

8. Tokarev, I.; Tokareva, I.; Minko, S. Gold-NanoparticleEnhanced Plasmonic Effects in a Responsive Polymer Gel. Adv. Mater. 2008, 20, 2730-2734.

9. Luzinov, I.; Minko, S.; Tsukruk, V. V. Responsive Brush Layers: From Tailored Gradients to Reversibly Assembled Nanoparticles. Soft Matter 2008, 4, 714-725.

10. Tokarev, I; Minko, S. Stimuli-Responsive Hydrogel Thin Films. Soft Matter 2009, 5, 511-524.

11. Mendes, P. M. Stimuli-Responsive Surfaces for Bioapplications. Chem. Soc. Rev. 2008, 37, 2512-2529.

12. Crowe, J. A.; Genzer, J. Creating Responsive Surfaces with Tailored Wettability Switching Kinetics and Reconstruction Reversibility. J. Am. Chem. Soc. 2005, 127, 17610-17611.

13. Singamaneni, S.; LeMieux, M. C.; Lang, H. P.; Gerber, Ch.; Lam, Y.; Zauscher, S.; Datskos, P. G.; Lavrik, N. V.; Jiang, H.; Naik, R. R.; et al. Bimaterial Microcantilevers As a Hybrid Sensing Platform. Adv. Mater. 2008, 20, 653-680.

14. Singamaneni, S.; McConney, M. E.; LeMieux, M. C.; Jiang, H.; Enlow, J. O.; Bunning, T. J.; Naik, R. R; Tsukruk, V. V. Polymer-Silicon Flexible Structures for Fast Chemical Vapor Detection. Adv. Mater. 2007, 19, 4248-4255.

15. Stuart, M. C.; Huck, W.; Genzer, J.; Müller, M.; Ober, C.; Stamm, M.; Sukhorukov, G.; Szleifer, I.; Tsukruk, V. V.; Urban, M.; et al. Emerging Applications of StimuliResponsive Polymer Materials. Nat. Mater. 2010, 9 , 101-113.

16. Senaratne, W.; Andruzzi, L.; Ober, C. K. Self-Assembled Monolayers and Polymer Brushes in Biotechnology: Current Applications and Future Perspectives. Biomacromolecules 2005, 6, 2427-2448.

17. Jhaveri, S. J.; Hynd, M. R.; Dowell-Mesfin, N.; Turner, J. N.; Shain, W.; Ober, C. K. Release of Nerve Growth Factor from HEMA Hydrogel-Coated Substrates and Its Effect on the Differentiation of Neural Cells. Biomacromolecules 2009, 10, 174-183.

18. Santer, S.; Kopyshev, A.; Donges, J.; Yang, H. K.; Ruhe, J. Dynamically Reconfigurable Polymer Films: Impact on Nanomotion. Adv. Mater. 2006, 18, 2359-2362.

19. Ayres, N.; Cyrus, C. D.; Brittain, W. J. Stimuli-Responsive Surfaces Using Polyampholyte Polymer Brushes Prepared via Atom Transfer Radical Polymerization. Langmuir 2007, 23, 3744-3749.

20. Kang, J.-H.; Moon, J. H.; Lee, S.-K.; Park, S.-G.; Jang, S. G.; Yang, S.; Yang, S.-M. Thermoresponsive Hydrogel Photonic Crystals by Three-Dimensional Holographic Lithography. Adv. Mater. 2008, 20, 3061-3065.

21. Julthongpiput, D.; Lin, Y. H.; Teng, J.; Zubarev, E. R.;
Tsukruk, V. V. Y-Shaped Amphiphilic Brushes with Switchable Micellar Surface Structures. J. Am. Chem. Soc. 2003, 125, 15912-15921.

22. Peleshanko, S.; Anderson, K. D.; Goodman, M.; Determan M. D.; Mallapragada, S. K.; Tsukruk, V. V. Thermoresponsive Reversible Behavior of Multistimuli Pluronic-Based Pentablock Copolymer at the Air-Water Interface. Langmuir 2007, 23, 25-30.

23. LeMieux, M. C.; Peleshanko, S.; Anderson, K. D.; Tsukruk, V. V. Adaptive Nanomechanical Response of Stratified Polymer Brush Structures. Langmuir 2007, 23, 265-273.

24. Sidorenko, A.; Krupenkin, T.; Taylor, A.; Fratzl, P.; Aizenberg, J. Reversible Switching of Hydrogel-Actuated Nanostructures into Complex Micropatterns. Science 2007, 315, 487-490.

25. Kloxin, A. M.; Kasko, A. M.; Salinas, C. N.; Anseth, K. S. Photodegradable Hydrogels for Dynamic Tuning of Physical and Chemical Properties. Science 2009, 324, 5963.

26. McConney, M. E.; Chen, N.; Lu, D.; Hu, H. A.; Coombs, S. Liu, C.; Tsukruk, V. V. Biologically Inspired Design of Hydrogel-Capped Hair Sensors for Enhanced Underwater Flow Detection. Soft Matter 2009, 5, 292-295.

27. Anderson, K. D.; Lu, D.; McConney, M.; Han, T.; Reneker, D.; Tsukruk, V. V. Hydrogel Microstructures Combined with Electrospun Fibers and Photopatterning for Shape and Modulus Control. Polymer 2008, 49, 5284-5293.

28. Bowden, N.; Brittain, S.; Evans, A. G.; Hutchinson, J. W.; Whitesides, G. M. Spontaneous Formation of Ordered Structures in Thin Films of Metals Supported on an Elastomeric Polymer. Nature 1998, 393, 146-149.

29. Efimenko, K.; Rackaitis, M.; Manias, E.; Vaziri, A.; Mahadevan, L.; Genzer, J. Nested Self-Similar Wrinkling Patterns in Skins. Nat. Mater. 2005, 4, 293-297.

30. Singamaneni, S.; Bertoldi, K.; Chang, S.; Jang, J. -H.; Thomas, E. L.; Boyce, M. C; Tsukruk, V. V. Instabilities and Pattern Transformation in Periodic, Porous Elastoplastic Solid Coatings. ACS Appl. Mater. Interfaces 2009, 1, 42-47.

31. Singamaneni, S.; Bertoldi, K.; Chang, S.; Jang, J. - H.; Young, S. L.; Thomas, E. L.; Boyce, M. C.; Tsukurk, V. V. Bifurcated Mechanical Behavior of Deformed Periodic Porous Solids. Adv. Funct. Mater. 2009, 19, 1426-1436.

32. Netzer, N. L.; Gunawidjaja, R.; Hiemstra, M.; Zhang, Q.; Tsukruk, V. V.; Jiang, C. Formation and Optical Properties of Compression-Induced Nanoscale Buckles on Silver Nanowires. ACS Nano 2009, 3, 1795-1802.

33. Gunawidjaja, R.; Ko, H.; Jiang, C.; Tsukruk, V. V. Buckling Behavior of Highly Oriented Silver Nanowires Encapsulated within LbL Film. Chem. Mater. 2007, 19, 2007-2015.

34. Mahadevan, L.; Rica, S. Self-Organized Origami. Science 2005, 307, 1740 .

35. Hiller, J.; Mendelsohn, J. D.; Rubner, M. F. Reversibly Erasable Nanoporous Antireflection Coatings from Polyelectrolyte Multilayers. Nat. Mater. 2002, 1, 59-63.

36. Stafford, C. M.; Harrison, C.; Beers, K. L.; Karim, A.; Amis, E. J.; VanLandingham, M. R.; Kim, H. C.; Volksen, W.; Miller, R. D.; Simonyi, E. E. A Buckling-Based Metrology for Measuring the Elastic Moduli of Polymeric Thin Films. Nat. Mater. 2004, 3, 545-550.

37. Jiang, C.; Singamaneni, S.; Merrick, E.; Tsukruk, V. V. Complex Buckling Instability Patterns of Nanomembranes with Encapsulated Gold Nanoparticle Arrays. Nano Lett. 2006, 6, 2254-2259.

38. Huang, J.; Juszkiewicz, M.; de Jeu, W. H.; Cerda, E.; Emrick T.; Menon, N.; Russell, T. P. Capillary Wrinkling of Floating Thin Polymer Films. Science 2007, 317, 650-653.

39. Chen, J. -T.; Zhang, M.; Russell, T. P. Instabilities in Nanoporous Media. Nano Lett. 2007, 7, 183-187.

40. Chan, E. P.; Smith, E. J.; Hayward, R. C.; Crosby, A. J. Surface Wrinkles for Smart Adhesion. Adv. Mater. 2008, 20, 711-716.

41. Genzer, J.; Groenewold, J. Soft Matter with Hard Skin: From Skin Wrinkles to Templating and Material Characterization. Soft Matter 2006, 2, 310-323. 
42. Shin, K.; Xiang, H.; Moon, S. I.; Kim, T.; McCarthy, T. J.; Russell, T. P. Curving and Frustrating Flatland. Science 2004, 306, 76.

43. Tanaka, T.; Sun, S.-T.; Hirokawa, Y.; Katayama, S.; Kucera, J.; Hirose, Y.; Amiya, T. Mechanical Instability of Gels at the Phase Transition. Nature 1987, 325, 796-798.

44. Hwa, T.; Kardar, M. Evolution of Surface Patterns on Swelling Gels. Phys. Rev. Lett. 1988, 61, 106-109.

45. Tanaka, H.; Tomita, H.; Takasu, A.; Hayashi, T.; Nishi, T. Morphological and Kinetic Evolution of Surface Patterns in Gels during the Swelling Process: Evidence of Dynamic Pattern Ordering. Phys. Rev. Lett. 1992, 68, 2794-2797.

46. Matsuo, E. S.; Tanaka, T. Patterns in Shrinking Gels. Nature 1992, 358, 482-485.

47. Mora, T.; Boudaoud, A. Buckling of Swelling Gels. Eur. Phys. J., E 2006, 20, 119-124.

48. Trujillo, V.; Kim, J.; Hayward, R. C. Creasing Instability of Surface-Attached Hydrogels. Soft Matter 2008, 4, 564-569.

49. Pocivavsek, L.; Dellsy, R.; Kern, A.; Johnson, S.; Lin, B.; Lee, K. Y. C.; Cerda, E. Stress and Fold Localization in Thin Elastic Membranes. Science 2008, 320, 912-916.

50. Lobkovsky, A.; Gentges, S.; Li, H.; Morse, D.; Witten, T. A Scaling Properties of Stretching Ridges in a Crumpled Elastic Sheet. Science 1995, 270, 1482-1485.

51. Vliegenthart, G. A.; Gompper, G. Forced Crumpling of SelfAvoiding Elastic Sheets. Nat. Mater. 2006, 5, 216-221.

52. Tallinen, T.; Åström, J. A.; Timonen, J. The Effect of Plasticity in Crumpling of Thin Sheets. Nat. Mater. 2009, 8, 25-29.

53. Singamaneni, S.; McConney, M. E.; Tsukruk V. V. Spontaneous Regular Folding in Confined Ultrathin Polymer Gels. Adv. Mater., published online January 4, 2010, http://dx.doi.org/10.1002/adma.200903052.

54. Orlov, M.; Tokarev, I.; Scholl, A.; Doran, A.; Minko, S. pHResponsive Thin Film Membranes from Poly(2-vinylpyridine): Water Vapor-Induced Formation of a Microporous Structure. Macromolecules 2007, 40, 2086-2091.

55. Roiter, Y.; Minko, S. AFM Single Molecule Experiments at the Solid-Liquid Interface: In Situ Conformation of Adsorbed Flexible Polyelectrolyte Chains. J. Am. Chem. Soc. 2005, 127, 15688-15689.

56. Chang, S.; Singamaneni, S.; Kharlampieva, E.; Young, S.; Tsukruk, V. V. Responsive Hybrid Nanotubes Composed of Block Copolymer and Gold Nanoparticles. Macromolecules 2009, 42, 5781-5785.

57. Malynych, S.; Luzinov, I.; Chumanov, G. Poly(vinyl pyridine) as a Universal Surface Modifier for Immobilization of Nanoparticles. J. Phys. Chem. B 2002, 106, 1280-1285.

58. Kozlovskaya, V.; Kharlampieva, E.; Chang, S.; Muhlbauer, R.; Tsukruk, V. V. pH-Responsive Layered Hydrogel Microcapsules as Gold Nanoreactors. Chem. Mater. 2009, 21, 2158-2167.

59. Singamaneni, S.; Kharlampieva, E.; Jang, J.-H.; McConney, M. E.; Jiang, H.; Bunning, T. J.; Thomas, E. L.; Tsukruk, V. V. Metallized Porous Interference Lithographic Microstructures via Biofunctionalization. Adv. Mater., published online January, 10, 2010, http://dx.doi.org/10.1002/adma.200902893.

60. Singamaneni, S.; Chang, S.; Jang, J. -H.; Davis, W.; Thomas, E. L.; Tsukruk, V. V. Mechanical Properties of 2D and 3D Polymer Microstructures Fabricated by Interference Lithography. Phys. Chem. Chem. Phys. 2008, 10, 4093-4105.

61. Ko, H.; Jiang, C.; Tsukruk, V. V. Encapsulating Nanoparticle Arrays into Layer-by-Layer Multilayers by Capillary Transfer Lithography. Chem. Mater. 2005, 17, 5489-5497.

62. Xu, G.; Bayly, P. V.; Taber, L. A. Residual Stress in the Adult Mouse Brain. Biomech. Model Mechanobiol. 2009, 8, 253-262.

63. Hamant, O.; Heisler, M. G.; Jönsson, H.; Krupinski, P.; Uyttewaal, M.; Bokov, P.; Corson, F.; Sahlin, P.; Boudaoud, A.; Meyerowitz, E. M.; Couder, Y.; Traas, J. Developmental Patterning by Mechanical Signals in Arabidopsis. Science 2008, 322, 1650-1655.
64. Dumais, J. Plant Morphogenesis: A Role for Mechanical Signals. Curr. Biol. 2009, 19, R207-R208.

65. Tsukruk, V. V.; Bliznyuk, V. N. Adhesive and Friction Forces between Chemically Modified Silicon and Silicon Nitride Surfaces. Langmuir 1998, 14, 446-455.

66. Jang, J.-H.; Ullal, C. K.; Choi, T.; Lemieux, M. C.; Tsukruk, V. V.; Thomas, E. L. 3D Polymer Microframes that Exploit Length-Scale-Dependent Mechanical Behavior. Adv. Mater. 2006, 18, 2123-2127.

67. Tsukruk, V. V. Scanning Probe Microscopy of Polymer Surfaces. Rubber Chem. Technol. 1997, 70, 430-475. 